\title{
Estimating Aerodynamic Coefficients from Uncertain Data of D-SEND Aircraft with Gaussian Process Regression*
}

\author{
Hidekazu KARINO, ${ }^{1) \dagger}$ Takehisa YAIRI, ${ }^{1)}$ Tetsujiro NINOMIYA, ${ }^{2)}$ and Koichi HoRI ${ }^{1)}$ \\ ${ }^{1)}$ Department of Aeronautics and Astronautics, The University of Tokyo, Tokyo 113-8656, Japan \\ ${ }^{2)}$ Aeronautical Technology Directorate, Japan Aerospace Exploration Agency, Mitaka, Tokyo 181-0015, Japan
}

\begin{abstract}
When simulating airflow we assume an ideal situation, however, flight test data includes measurement noise when actually conducted. Therefore, it is difficult to compare simulation data with flight test data without considering uncertainty. First, we applied the Noisy Input Gaussian Process (NIGP), which can utilize uncertain inputs to estimate aerodynamic coefficients with confidence intervals to an aircraft's simulation data. This enabled us to verify the effectiveness of NIGP. We then applied NIGP to the aircraft's real flight data and compared them with aerodynamic tables based on wind tunnel testing and CFD. We found that the lift coefficient estimated using the flight test data did not contradict that obtained using simulation data, while the drag coefficient estimated using the flight test data was smaller than that obtained using the simulation data.
\end{abstract}

Key Words: Flight Control, Flight Dynamics, Flight Testing

\section{Nomenclature}

$$
\begin{aligned}
C_{L} & : \text { lift force coefficient } \\
C_{D} & \text { drag force coefficient } \\
\alpha & : \text { angle of attack, deg } \\
\beta & \text { sideslip angle, deg } \\
M: & \text { Mach number } \\
m: & \text { mass of D-SEND, } \mathrm{kg} \\
Q: & \text { dynamic pressure, } \mathrm{Pa} \\
S: & \text { wing area, } \mathrm{m}^{2} \\
L: & \text { lift force, } \mathrm{N} \\
D: & \text { drag force, } \mathrm{N} \\
\delta_{e}: & \text { angle of stabilizer, deg } \\
\delta_{r}: & \text { angle of rudder, deg } \\
\delta_{a}: & \text { angle of aileron, deg } \\
a_{x}: & X \text {-axis acceleration, } \mathrm{m} / \mathrm{s}^{2} \\
a_{z}: & Z \text {-axis acceleration, } \mathrm{m} / \mathrm{s}^{2} \\
\mathbb{E}: & \text { expected value } \\
\mathbb{V}: & \text { variance }
\end{aligned}
$$

$\mathcal{N}\left(\mu, \sigma^{2}\right)$ : normal distribution with the mean $\mu$ and the variance $\sigma^{2}$

Subscripts

$$
\begin{aligned}
& \text { s: } \text { stabilizer } \\
& \text { r: } \text { rudder } \\
& \text { sa: } \text { aileron } \\
& \text { flex: } \text { aeroelasticity } \\
& \text { intl: inertia }
\end{aligned}
$$

\section{Introduction}

It is important to estimate aerodynamic coefficients accurately. When designing an aircraft's flight controllers we

C 2020 The Japan Society for Aeronautical and Space Sciences *Received 25 November 2019; final revision received 7 June 2020; accepted for publication 15 June 2020.

†Corresponding author, hidekazu-karino-main@g.ecc.u-tokyo.ac.jp need to know the aerodynamic forces affecting the aircraft to guarantee its safety, and when calculating fuel consumption, we need to know drag precisely. In conceptual design phase analytical methods, the geometrical details of an aircraft can be given through roughly estimating aerodynamic coefficients. However, in the detailed design phase, there are mainly three methods to estimate aerodynamic coefficients: wind tunnel testing, CFD and flight tests, and those three methods each have pros and cons. Unlike wind tunnel testing and flight tests, CFD can be carried out with relatively low cost (both monetary/labor cost), but computer simulation is based on the input of physical laws and has parameters. By comparing simulation data with flight test data, it can be confirmed that simulation data match real phenomena and that parameters are well-tuned. However, flight test data is gathered via sensors that have measurement noise, so flight test data and simulation data do not match exactly. Therefore, without considering uncertainty, it is not possible to figure out if computer simulation is accurate.

The aerodynamic coefficients of an aircraft are estimated from flight data using various computational methods and wind tunnel testing. ${ }^{1,2)}$ In previous studies, conventional methods are applied using actual flight data. As the development of the machine learning field has advanced, data-driven approaches have become more sophisticated. Gaussian Process Regression (GPR) is one of these approaches. Chati and Balakrishnan $^{3)}$ estimated the takeoff weight of an aircraft using GPR, and it shows the efficacy of GPR to model aircraft parameters using flight test data. Hemakumara and Sukkar$\mathrm{ieh}^{4)}$ applied GPR to generate an aerodynamic model using flight data by applying local and global approximations obtained with GPs. This method reduces the computational burden of a GP applied to large-scale datasets, and shows good prediction performance compared to measured aerodynamic coefficients. Kumar and Ghosh ${ }^{5)}$ applied GPR for nonlinear aerodynamic modeling using flight test data and compared 


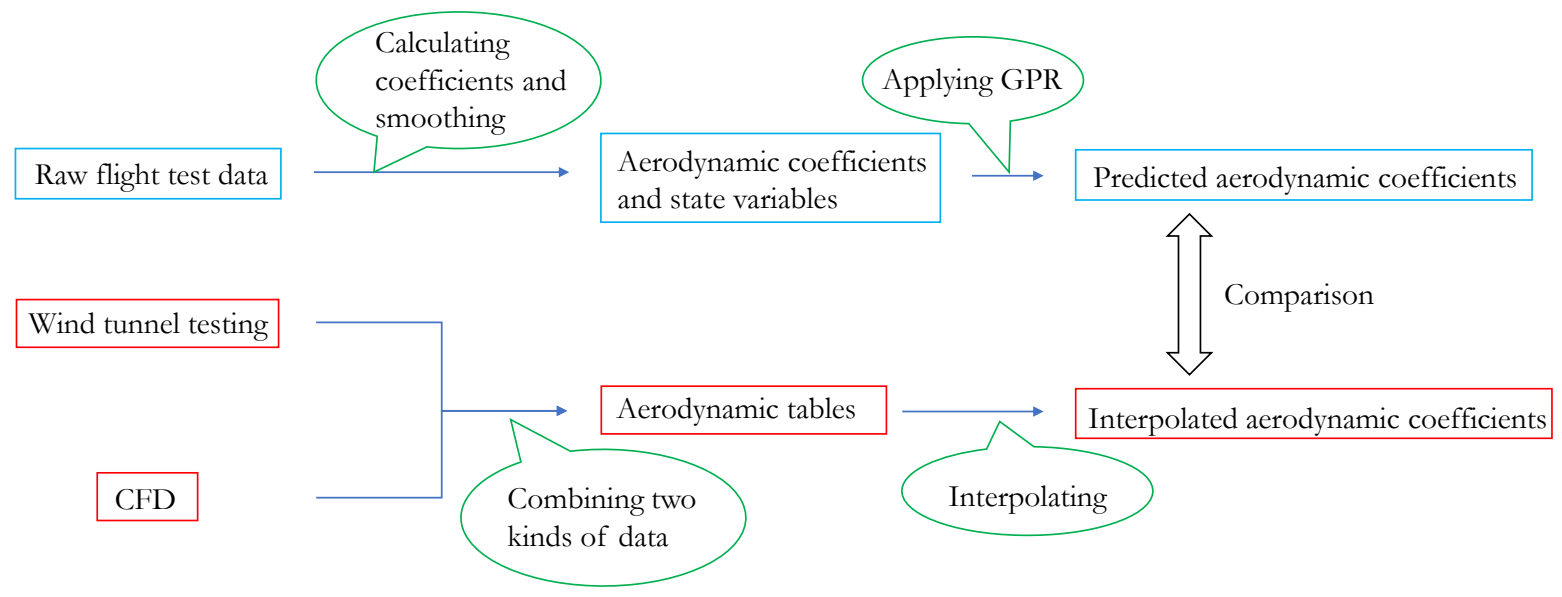

Fig. 1. Procedure of comparing flight test data with aerodynamic tables.

Predicted aerodynamic coefficients are calculated using GPR from smoothed flight test data and aerodynamic tables (prepared by experts in aerodynamics and based on wind tunnel testing and CFD data) are interpolated, and both kinds of coefficients are compared.

the results to the maximum-likelihood estimation (MLE) method. This approach shows a good approximation of both measured aerodynamic coefficients and MLE predictions. Although these studies ${ }^{4,5)}$ applied the GPR method to generate an aerodynamic model from actual flight test data, there was no comparison made with other models generated from wind tunnel testing or CFD. We applied GPR to flight test data of the Drop test for Simplified Evaluation of Non-symmetrically Distributed sonic boom (D-SEND) project so that we could compare estimated aerodynamic coefficients of flight test data with those of aerodynamic tables. The procedure for comparing flight test data with aerodynamic tables is shown in Fig. 1.

This paper has the following contributions: 1. Development of a GPR-based method to model aerodynamic coefficients that are confirmed to show good performance through numerical simulation. 2. Comparison of interval estimation results of the method presented with aerodynamic tables, indicating that the aerodynamic table have large error.

The remainder of this paper is organized as follows: Section 2 describes the reason why we used GPR for estimating aerodynamic coefficients from flight test data. Section 3 introduces the algorithm of Noisy Input GPR, the problem setting and dataset for estimating aerodynamic coefficients. Section 4 compares aerodynamic coefficients estimated using simulation data and real flight test data. Section 5 discusses estimated drag coefficients of flight test data and the effect of measurement noise of Noisy Input GPR input variables. The conclusions are provided in Section 6.

\section{Why We Used GPR}

\subsection{Three kinds of data obtained from the D-SEND project}

D-SEND is a project conducted by the Japan Aerospace Exploration Agency (JAXA) to demonstrate and evaluate the designing method of low sonic boom aircraft. At the Swedish Esrange experiment site, the D-SEND aircraft suspended by a balloon was separated at an altitude of $30 \mathrm{~km}$ and flew to one of the sonic boom measurement points. The experiment was a success and not only sonic boom sound data, but also flight test data of the D-SEND aircraft were gathered. Flight test data had sensor data such as $\alpha$, $M$ and $Q$. Using such data, we also calculated aerodynamic coefficients like $C_{L}$ and $C_{D}$.

Before the flight test was conducted, CFD and wind tunnel testing were also conducted, and based on the data of those experiments, aerodynamic tables were generated. Aerodynamic tables are databases containing each term of aerodynamic forces, such as $\mathrm{d} C_{L \mathrm{~s}}$ representing lift force generated by the stabilizer. In aerodynamic tables, aerodynamic forces are defined as the summation of these terms.

Moreover, after the D-SEND experiment, computer simulation of the aircraft's flight using aerodynamic tables was carried out. In this simulation, aerodynamic forces were calculated using aerodynamic tables, and control commands based on control laws were output to the control surface of the D-SEND aircraft. When calculating aerodynamic forces and control commands, measurement noise was considered, and both noise-free data and noisy data were left. If predicted confidence intervals of aerodynamic coefficients applying GPR using noisy data fit those using noise-free data, this would prove that our method is effective. This procedure is shown in Fig. 2.

\subsection{Aerodynamic tables}

Aerodynamic coefficients are not constant values, but functions from variables like $\alpha$ and $M$ to coefficients like $C_{L}$ and $C_{D}$. Aerodynamic tables define relations between these discrete variables and aerodynamic coefficients. Experts in aerodynamics made aerodynamic tables using two kinds of data: wind tunnel experiments and CFD.

In aerodynamic tables, $C_{L}$ and $C_{D}$ are defined as follows.

$$
\begin{aligned}
C_{L}= & C_{L \text { basic }}+\mathrm{d} C_{L \mathrm{~s}}+\mathrm{d} C_{L \mathrm{r}} \\
& +\mathrm{d} C_{L \text { sa }}+\mathrm{d} C_{L \text { flex }}+\mathrm{d} C_{L \text { intl }} \\
C_{D}= & C_{D \text { basic }}+\mathrm{d} C_{D \mathrm{~s}}+\mathrm{d} C_{D \mathrm{r}} \\
& +\mathrm{d} C_{D \text { sa }}+\mathrm{d} C_{D \text { flex }}+\mathrm{d} C_{D \text { intl }}
\end{aligned}
$$




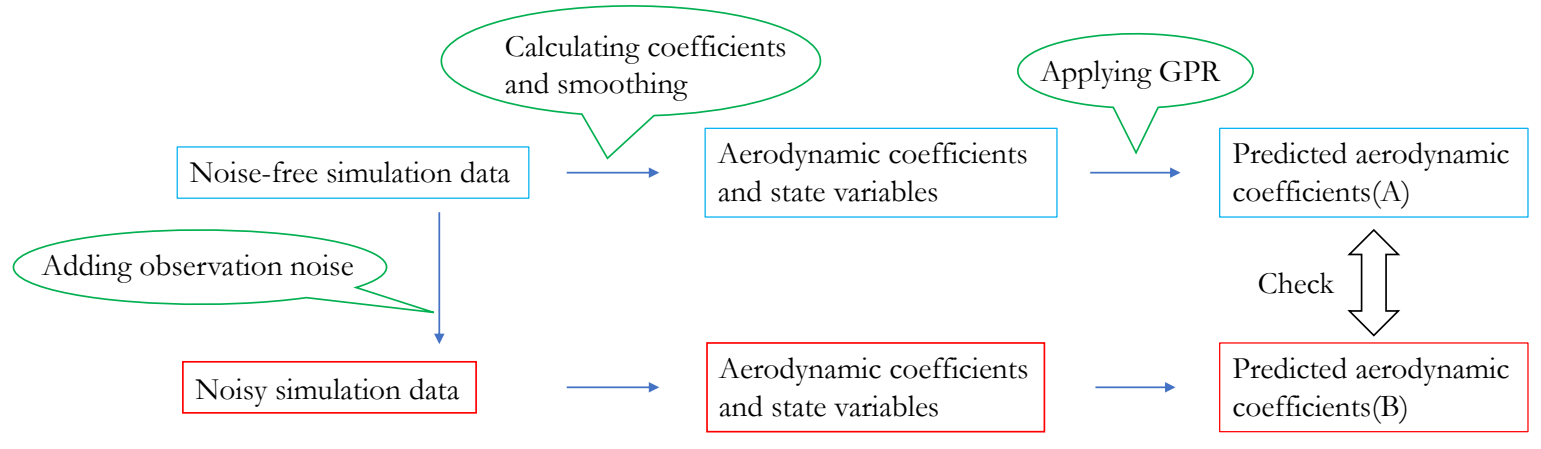

Fig. 2. Usage of noise-free simulation data.

By applying GPR to noise-free data, we obtain predicted aerodynamic coefficients (A); and by applying GPR to noisy data, we obtain predicted aerodynamic coefficients (B). We checked to see if the confidence intervals of B included A.

Table 1. Arguments of each element.

\begin{tabular}{lcccccccc}
\hline Subscript & $\alpha$ & $\beta$ & $M$ & $\delta_{e}$ & $\delta_{r}$ & $\delta_{a}$ & $Q$ & $a_{z}$ \\
\hline basic & $\checkmark$ & $\checkmark$ & $\checkmark$ & & & & & \\
s & $\checkmark$ & $\checkmark$ & $\checkmark$ & $\checkmark$ & & & & \\
r & $\checkmark$ & $\checkmark$ & $\checkmark$ & & $\checkmark$ & & & \\
sa & $\checkmark$ & $\checkmark$ & $\checkmark$ & $\mathfrak{V}^{*}$ & & $\checkmark$ & & \\
flex & $\mathfrak{J}^{\dagger}$ & & $\checkmark$ & & & & $\checkmark$ & \\
intl & $\mathfrak{J}^{\ddagger}$ & & $\checkmark$ & & & & & $\mathfrak{S}^{\S}$ \\
\hline
\end{tabular}

*In this case, defined every $10 \mathrm{deg}$.

${ }^{\dagger}$ In this case, defined every $2 \mathrm{deg}$.

${ }^{\ddagger}$ In this case, defined every $2 \mathrm{deg}$.

${ }^{\S}$ In this case, the argument is the absolute value of $a_{z}$.

Each element of Eqs. (1) and (2) is defined in the tables. They are also functions with some arguments, as shown in Table 1.

In Table $1, \alpha$ is defined every $1 \mathrm{deg}$ from -10 to $15, \beta$ by 0 and $5 \mathrm{deg}, M$ by either of $(0.6,0.8,0.9,0.95,1.05,1.1$, $1.2,1.4), \delta_{e}$ every $5 \mathrm{deg}, \delta_{r}$ every $10 \mathrm{deg}, \delta_{a} 5 \mathrm{deg}, Q$ every $10 \mathrm{kPa}$ and $a_{z}$ every $10 \mathrm{~m} / \mathrm{s}^{2}$ unless otherwise noted. Compared with the arguments of the tables, the grid size of each state variable of flight test data is so small that it is necessary to interpolate the values of the aerodynamic tables for comparing flight test data with the tables. In this paper, the values of the aerodynamic tables were interpolated linearly for each state variable.

Aerodynamic tables take all state variables as arguments. However, because the term of $C_{L \text { basic }}$ is much larger than the other terms and all terms take $\alpha$ and $M$ as inputs, we made use of only $\alpha$ and $M$ as inputs for GPR.

\subsection{Merit of Noisy Input GPR}

Previously, flight test data and aerodynamic simulation data were compared directly by applying point estimation using algorithms like the least squares method, but this has some problems.

First, when estimating aerodynamic coefficients using the least squares method, the problem of multicollinearity often occurs. ${ }^{6)}$ Multicollinearity refers to predictors that are correlated with other predictors in the linear regression model. This is because the inverse matrix, which has correlated values and does not have full rank, is unstable. The input values ( $\alpha$ and $\delta_{e}$ ) are correlated, so it is hard to avoid multicollinear- ity when using the least squares method.

Second, flight test data has sensor values and aerodynamic coefficients are calculated using relational expressions like Eqs. (20) and (21) that include these sensor values (aerodynamic coefficients cannot be measured directly). Therefore, not only state variables like $\alpha$ and $M$, but also values like $C_{L}$ and $C_{D}$ have measurement noise. The conventional method cannot deal with this input uncertainty.

On the other hand, Noisy Input GPR can handle these problems because it does not need an inverse matrix. Therefore, it can avoid multicollinearity, and by expanding standard GPR, we can cope with the uncertainty of input. As a result, we used the Noisy Input Gaussian Process (NIGP) to estimate aerodynamic coefficients.

\section{Algorithm of Noisy Input GPR}

\subsection{Overview of GPR}

GPR is an algorithm that deals with supervised learning, which is the problem of learning the relationship between input and continuous output. ${ }^{7)}$ It is a non-linear regression method and assumes output follows multivariate Gaussian distribution, so the prediction has uncertainty information (i.e., the variance in Gaussian distribution). This was also developed as Kriging in the field of statistics to estimate the most likely distribution of gold from a few samples ${ }^{8)}$ and now widely used in the field of machine learning to optimize parameters, ${ }^{9)}$ to model biosystems, ${ }^{10)}$ and so on.

\subsection{GPR}

Standard GPR is derived as follows.

Let $\boldsymbol{x}$ and $y$ be a pair of observed values, where $\boldsymbol{x}$ is a dimensional input like $\boldsymbol{x}=(\alpha, M)$, and $y$ is the corresponding scalar output like $C_{L}$ and $C_{D}$. We assume that measured $y$ is the sum of Gaussian measurement noise and actual value $\tilde{y}$. Measurement noise is independent of $\tilde{y}$ and is caused by sensors (not by atmospheric turbulence).

$$
\begin{aligned}
y & =\tilde{y}+\epsilon_{y} \\
\epsilon_{y} & \sim \mathcal{N}\left(0, \sigma_{y}^{2}\right)
\end{aligned}
$$

Suppose we have $n$ measurements and the $i$-th measurement is denoted by $\boldsymbol{x}_{i}$ and $y_{i}$. By assuming that $\tilde{y}$ follows the linear model $f(x)=\sum_{i} w_{i} \phi_{i}(x)$, where $w_{i}$ is weight 
and $\phi_{i}(x)$ is the basis function, and $w_{i}$ follows Gaussian distribution, $f(x)$ follows Gaussian distribution too. Gaussian distribution is described using two statistical measurements, mean and variance, and they can be calculated using the kernel function $k(a, b)$. The kernel function has two arguments and returns a scalar value.

$$
\begin{aligned}
& \tilde{y}_{i}=f\left(\boldsymbol{x}_{i}\right) \\
& \mathbb{E}[f(\boldsymbol{x})]=0 \\
& \mathbb{V}[f(\boldsymbol{x})]=K \\
& K_{i j}=k\left(x_{i}, x_{j}\right)
\end{aligned}
$$

Since $\tilde{y}$ and $\epsilon_{y}$ are independent, variance in $y$ is just the sum of variance in $\tilde{y}$ and variance in $\epsilon_{y}$.

$$
\mathbb{V}[y]=K+\sigma_{y}^{2} I
$$

\subsection{Considering noisy input}

In the previous section, we considered only output noise, but input is often corrupted as well. NIGP, ${ }^{11)}$ which can manage input uncertainty, is derived as follows.

Suppose input $x$ is the sum of true value $\tilde{x}$ and noise $\epsilon_{x}$ following Gaussian distribution.

$$
\begin{aligned}
x & =\tilde{x}+\epsilon_{x} \\
\epsilon_{x} & \sim \mathcal{N}\left(0 \sigma_{x}^{2}\right) \\
y & =f(\tilde{x})+\epsilon_{y}=f\left(x-\epsilon_{x}\right)+\epsilon_{y}
\end{aligned}
$$

The posterior distribution based on Eq. (11) is intractable, so we consider Taylor expansion.

$$
f\left(x-\epsilon_{x}\right)=f(x)-\epsilon_{x} \frac{\partial f}{\partial x}+\cdots
$$

The derivative of the GP is another GP, and considering distribution is needed, this exact treatment makes little difference. ${ }^{11)}$ Therefore, we consider only the derivative of the mean function of GP, which we denote as $\partial_{f}$. By expanding up to the first-order terms, we get a linear model with the input noise.

$$
y=f(x)-\epsilon_{x} \partial_{f}+\epsilon_{y}
$$

Since $f(x), \partial_{f}$ and $\epsilon_{y}$ are independent, the variance in $y$ is only the sum of each variance.

$$
\mathbb{V}[y]=K+\nabla f \Sigma_{x} \nabla f^{\mathrm{T}}+\Sigma_{y}=C
$$

where, $\nabla f=\left(\partial_{f}\left(x_{1}\right), \partial_{f}\left(x_{2}\right) \cdots \partial_{f}\left(x_{n}\right)\right)^{\mathrm{T}}$ and $\Sigma_{x}$ and $\Sigma_{y}$ are scalar matrices whose all on-diagonal elements are $\sigma_{x}^{2}$ and $\sigma_{y}^{2}$, respectively.

\subsection{Prediction}

Let the number of training data be $n$, that of test data be $m$ and input variables of test data be $\boldsymbol{x}_{m}$. The simultaneous distribution of training data and test data follows Gaussian distribution, the covariance matrix $C_{n+m}$ of which is calculated using Eq. (15).

$$
C_{n+m}=\left(\begin{array}{ll}
C_{n} & k \\
k^{\mathrm{T}} & C_{m}
\end{array}\right),
$$

where, $C_{n}$ and $C_{m}$ are the $n \times n$ and $m \times m$ covariance ma- trices of the training and test data, as shown in Eq. (14), and $k$ is a matrix whose element is $k\left(\boldsymbol{x}_{i}, \boldsymbol{x}_{j}\right)(i=1,2, \cdots, n$, $j=1,2, \cdots m)$.

The conditional probability distribution when $n$ observed values are obtained is a Gaussian distribution whose mean and covariance can be calculated using Eqs. (16) and (17), respectively.

$$
\begin{aligned}
\mu\left(\boldsymbol{x}_{m}\right) & =k^{\mathrm{T}} C_{n}{ }^{-1} \boldsymbol{t}_{n} \\
\sigma^{2}\left(\boldsymbol{x}_{m}\right) & =C_{m}-k^{\mathrm{T}} C_{n}{ }^{-1} k
\end{aligned}
$$

\subsection{Estimating measurement noise of aerodynamic coefficients}

Although the variance in measurement noise $\sigma_{y}^{2}$ is necessary for applying NIGP to flight test data, it is impossible to calculate it directly. Therefore, we estimated the variance in $C_{L}$ and $C_{D}$ by adding errors that are randomly sampled to each variable.

$C_{L}$ and $C_{D}$ are defined by Eqs. (20) and (21), respectively.

$$
\begin{aligned}
L & =m\left(a_{x} \sin \alpha-a_{z} \cos \alpha\right) \\
D & =-m\left(a_{x} \cos \alpha+a_{z} \sin \alpha\right) \\
C_{L} & =\frac{L}{Q S}=\frac{m}{S} \frac{a_{x} \sin \alpha-a_{z} \cos \alpha}{Q} \\
C_{D} & =\frac{D}{Q S}=\frac{-m}{S} \frac{a_{x} \cos \alpha+a_{z} \sin \alpha}{Q}
\end{aligned}
$$

The sensor errors are given by sensor specification (i.e., described in the report of D-SEND's flight simulation model ${ }^{12)}$ ) depending on the altitude and $M$. We considered the measurement noise of $a_{x}, a_{z}, \alpha$ and $Q$.

Suppose measured $V, a$ and $\alpha$ have sensor measurement noise. In each trial, we generate random values from the Gaussian distribution whose parameters are set by sensor specifications and subtract them from observed values, and the remainder is thought to be the true value. By doing this trial many times and calculating aerodynamic coefficients using Eqs. (20) and (21), the distributions of $C_{L}$ and $C_{D}$ are obtained. Furthermore, by fitting these distributions to Gaussian distribution, we obtain the estimated measurement noise parameters of the aerodynamic coefficient $\hat{\sigma}_{y}$. When optimizing parameters of NIGP, we fixed the measurement noise parameters of $C_{L}\left(\sigma_{y}\right)$ and $C_{D}\left(\sigma_{y}\right)$ to $\hat{\sigma}_{y}$.

\subsection{Parameter optimization}

In GPR, the posterior distribution obtained is Gaussian distribution, and we can evaluate the model by calculating log-likelihood, as shown in Eq. (22).

$$
\begin{aligned}
\log p(\boldsymbol{y} \mid \boldsymbol{x})= & -\frac{1}{2} \boldsymbol{y}^{\mathrm{T}}\left(K+\sigma_{y}^{2} I\right)^{-1} \boldsymbol{y}-\frac{1}{2}\left\|K+\sigma_{y}^{2} I\right\| \\
& -\frac{n}{2} \log 2 \pi
\end{aligned}
$$

First, we explored parameters by grid search and drew contour lines. With $\theta_{0}$ of the radial basis function in Eq. (23) fixed, the contour lines are shown in Fig. 5 in the Appendix. This plot seems to be unimodal; in other words, it has a single highest value in this parameter space. 
Since grid search requires a large amount of time, time grows exponentially based on input dimensions and contour lines do not seem to be multimodal, we search parameters by space partitioning. Space partitioning is the process of dividing parameter space into regions that do not overlap. For example, when we search parameters $l_{a}$ and $l_{b}$ from 0.0 to 1.0 , respectively, we split each parameter space into $h$ (e.g., $[0.0,0.5,1.0](h=2))$ and calculate log-likelihood at the center of each parameter space (e.g., $\left(l_{a}, l_{b}\right)=(0.25,0.25)$, $(0.25,0.75)$ and so on). After finding the highest point (e.g., $\left.\left(l_{a}, l_{b}\right)=(0.25,0.25)\right)$, we divide the parameter space as $l_{a}=[0,0.25,0.5]$ and calculate the log-likelihood at several points.

\subsection{Problem setting}

As we mentioned in Section 2.2, we set the input variables of GPR as $\alpha$ and $M$ to learn $C_{L}$ and $C_{D}$ (i.e., during D-SEND aircraft flight, the pitching moment was nearly zero, so we did not learn $C_{m}$ ). We chose radial basis function Eq. (23) as the kernel function because it can manage continuous values and it is a popular kernel function used in many kernelized learning algorithms.

$$
k\left(\boldsymbol{x}_{i}, \boldsymbol{x}_{j}\right)=\theta_{0} \exp \left\{-\frac{1}{2} \sum_{d}^{D}\left(\frac{x_{i, d}-x_{j, d}}{l_{d}}\right)^{2}\right\}
$$

In NIGP, there are several parameters (i.e., $\sigma_{x}, \sigma_{y}, \theta_{0}, l_{d}$ ), and they are optimized to maximize log-likelihood. As for $\sigma_{y}$, however, it is determined by measurement noise and we should not set it freely (i.e., $\sigma_{y}$ cannot be set to too small of a value, such as " 0 " because, if this is done, log-likelihood becomes larger). Moreover, $\sigma_{x}$ is assumed to follow a Gaussian distribution whose parameters are given by sensor specifications, so there are $D+1$ parameters $\left(\theta_{0}\right.$ and $l_{d}$ s) left to optimize.

\subsection{Datasets}

We estimated aerodynamic coefficients $\left(C_{L}\right.$ and $\left.C_{D}\right)$ by applying NIGP to two input variables ( $\alpha$ and $M$ ). Three kinds of data (i.e., flight test, noisy simulation and noise-free simulation) are shown in Figs. 6-8 in the Appendix. Variables like $\alpha$ and $M$ are real sensor data in the flight test data and virtual values in the simulation data, and aerodynamic coefficients like $C_{L}$ and $C_{D}$ are calculated using Eqs. (20) and (21), respectively. Both datasets contain input noise, and the simulation data has noise-free data too. If aerodynamic coefficients of noise-free data are within the confidence intervals of predicted distribution using noisy simulation data, NIGP would prove to be effective for estimating aerodynamic coefficients.

\section{Results}

\subsection{Estimated aerodynamic coefficients using simula- tion data}

Estimated $C_{L}$ and $C_{D}$ using noisy simulation data are shown in Fig. 3.

Both $C_{L}$ and $C_{D}$, the true values of noise-free simulation data, are within confidence intervals of predicted Gaussian distribution of NIGP even though predicted aerodynamic co-

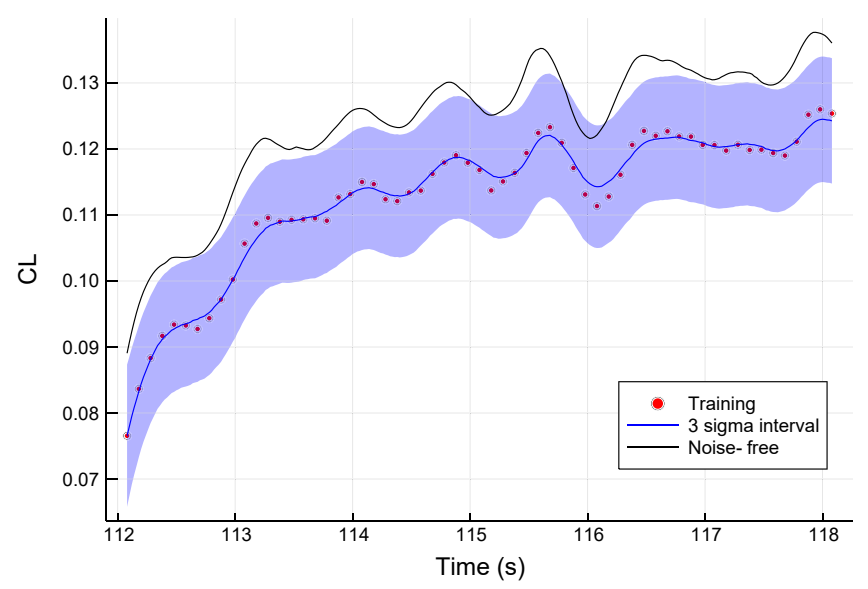

(a) Predicted $C_{L}$ using simulation data

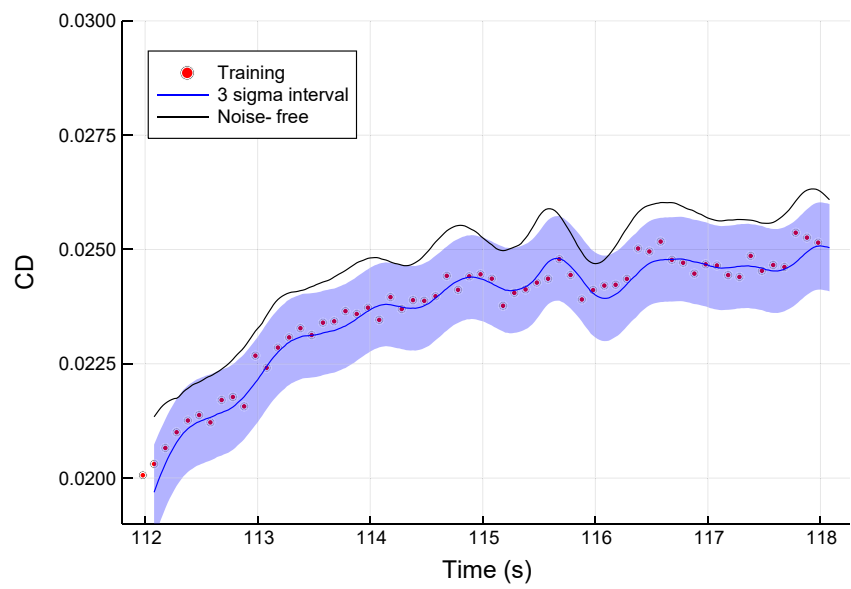

(b) Predicted $C_{D}$ using simulation data

Fig. 3. Predicted aerodynamic coefficients using simulation data. The blue area is the $3 \sigma$ interval of posterior Gaussian distribution, red points are the training data and black lines are the coefficients of noise-free data; namely, the true values of aerodynamic coefficients.

efficients have biases caused by measurement noise.

\subsection{Estimated aerodynamic coefficients using flight test data}

Estimated $C_{L}$ and $C_{D}$ using flight test data are shown in Fig. 4.

The $C_{L}$ of aerodynamic tables is within $3 \sigma$ intervals of the predicted Gaussian distribution while the $C_{D}$ is not within the $3 \sigma$ intervals.

\section{Discussion}

\subsection{Estimated drag coefficient of flight test data}

Figure 3 shows that estimated confidence intervals of both $C_{L}$ and $C_{D}$ from noisy simulation data include those from noise-free simulation data. This result indicates that, even if the input is corrupted, NIGP can predict intervals containing true values.

Figure 4(b) shows that the $C_{D}$ of aerodynamic tables is not within the $3 \sigma$ intervals of the estimated $C_{D}$ obtained from the D-SEND aircraft's test data. It was reported that the calculated $C_{D}$ from the flight test data was not within the $3 \sigma$ in- 


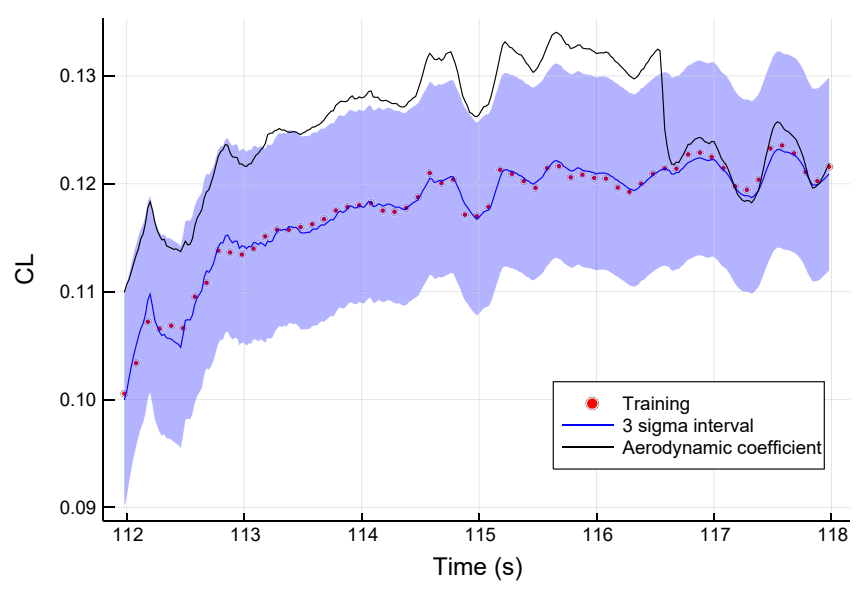

(a) Predicted $C_{L}$ using flight test data

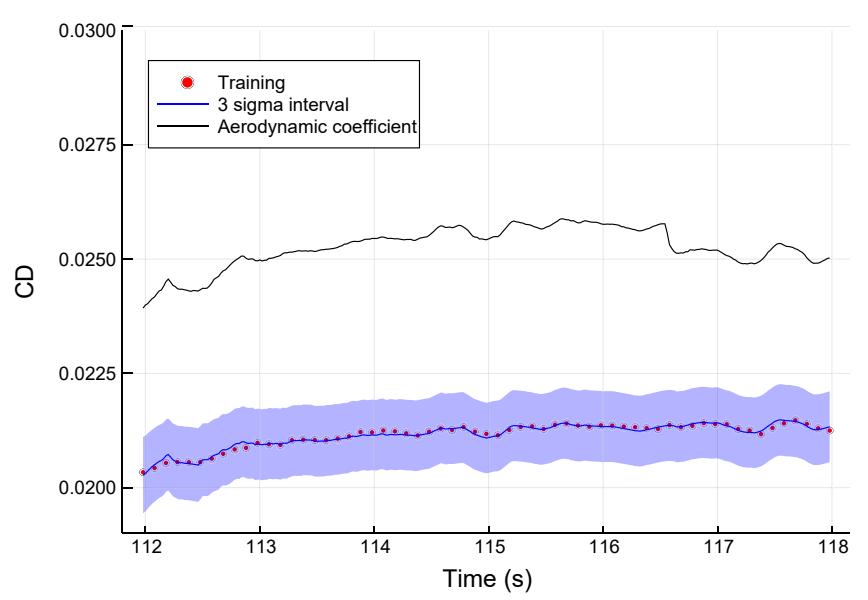

(b) Predicted $C_{D}$ using flight test data

Fig. 4. Predicted aerodynamic coefficients using flight test data.

The blue area shows the $3 \sigma$ intervals of posterior Gaussian distribution, red points are the training data and black lines are the aerodynamic coefficients of aerodynamic tables under the same conditions.

Table 2. Difference of $\sigma_{y}$ and $\sigma_{p}$ ( $\sigma$ of predicted Gaussian distribution).

\begin{tabular}{lll}
\hline & \multicolumn{1}{c}{$\sigma_{y}$} & \multicolumn{1}{c}{$\sigma_{p}$} \\
\hline$C_{L}$ & 0.0029 & 0.0030 \\
$C_{D}$ & 0.00025 & 0.00026 \\
\hline
\end{tabular}

tervals of the aerodynamic error model in the design phase. ${ }^{13)}$ The fact that the $C_{D}$ of aerodynamic tables was not within the $3 \sigma$ intervals of predicted NIGP distribution confirms that aerodynamic simulation in the design phase and under actual flight test conditions were different.

\subsection{Effect of input variable measurement noise}

We calculated $\sigma_{y}$ and $\sigma_{p}$ (i.e., $\sigma$ of predicted NIGP Gaussian distribution using flight test data), and they are shown in Table 2.

This result indicates that input noise has less influence on $\sigma_{p}$ than the measurement noise of aerodynamic coefficients. Therefore, to reduce the uncertainty of aerodynamic coefficients, more precise measurement sensors are required because $\sigma_{y}$ is estimated using Eqs. (20) and (21).

\section{Conclusion}

In this study we applied NIGP to the noisy simulation data and real flight test data of the D-SEND aircraft. NIGP was verified by comparing estimated aerodynamic coefficients from noisy simulation data with those from noise-free simulation data. The result of applying NIGP to the real flight test data showed that the estimated $C_{L}$ did not contradict with that listed in aerodynamic tables, while the estimated $C_{D}$ was much smaller than that listed in aerodynamic tables. This indicates that aerodynamic tables have larger $C_{D}$ values than actual values measured. This is consistent with the report that calculated $C_{D}$ from flight test data was not within the $3 \sigma$ intervals of the aerodynamic error model in the design phase.

We used D-SEND flight test data, but the flight test data was collected only once. Applying NIGP to other aircraft undergoing multiple flight tests is a subject for future work.

\section{References}

1) Jategaonkar, R.: Flight Vehicle System Identification: A Time Domain Methodology, Vol. 216, AIAA, 2006.

2) Klein, V. and Morelli, E.: Aircraft System Identification: Theory and Practice, AIAA, 2006.

3) Chati, Y. and Balakrishnan, H.: Modeling of Aircraft Takeoff Weight Using Gaussian Processes, J. Air Transportation, 26 (2018), pp. 1-10.

4) Hemakumara, P. and Sukkarieh, S.: UAV Parameter Estimation with Multi-output Local and Global Gaussian Process Approximations, 2013 IEEE International Conference on Robotics and Automation, 2013, pp. 5402-5408.

5) Kumar, A. and Ghosh, A. K.: GPR-based Novel Approach for Nonlinear Aerodynamic Modelling from Flight Data, Aeronautical J., 123, 1259 (2019), pp. 79-92.

6) Kobayashi, O.: Estimation of Aerodynamic Characteristics from Flight Test Data (2nd Report), J. Jpn. Soc. Aeronaut. Space Sci., 40, 466 (1992), pp. 617-626.

7) Rasmussen, C. E. and Williams, C. K. I.: Gaussian Process for Machine Learning, The MIT Press, 2006.

8) Krige, D. G.: A Statistical Approach to Some Mine Valuation and Allied Problems on the Witwatersrand, J. Southern African Institute of Mining and Metallurgy, 52 (1951), pp. 119-139.

9) Snoek, J., Larochelle, H., and Adams, R. P.: Practical Bayesian Optimization of Machine Learning Algorithms, Proceedings of the 25th International Conference on Neural Information Processing Systems, Vol. 2, NIPS'12, Red Hook, NY, USA, Curran Associates Inc, 2012, pp. 2951-2959.

10) Ažman, K. and Kocijan, J.: Application of Gaussian Processes for Black-box Modelling of Biosystems, ISA Trans., 46, 4 (2007), pp. 443-457.

11) McHutchon, A. and Rasmussen, C. E.: Gaussian Process Training with Input Noise, Advances in Neural Information Processing Systems 24 (NIPS 2011), pp. 1341-1349.

12) Kawaguchi, J., Suzuki, H., Ninomiya, T., and Tomita, H.: Report on the Flight Simulation Model of Flight Demonstration of Low Sonic Boom Design Concept Phase ii (D-SEND\#2), JAXA-RR-17-006, 2018.

13) Ninomiya, T., Suzuki, H., and Kawaguchi, J.: Dynamic Inversion Controller Design for Balloon-launched Supersonic Aircraft, Trans. Jpn. Soc. Aeronaut. Space Sci., 61 (2018), pp. 248-257. 


\section{Appendix}

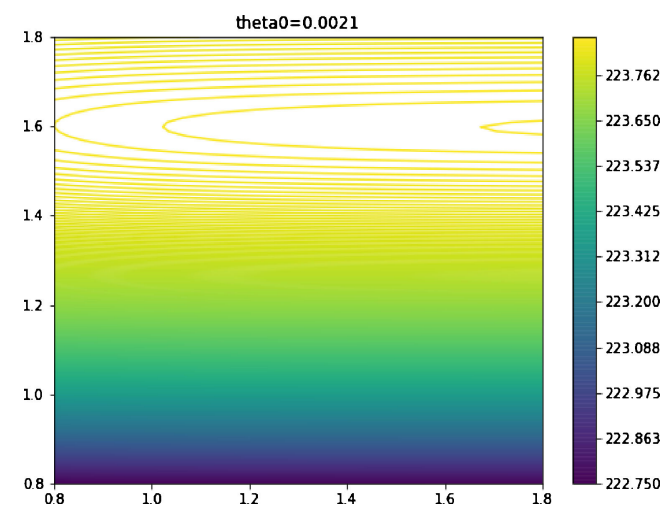

Fig. 5. Contour plot of log-likelihood.

\section{A. Contour plots of log-likelihood}

Fig.

\section{B. Datasets}

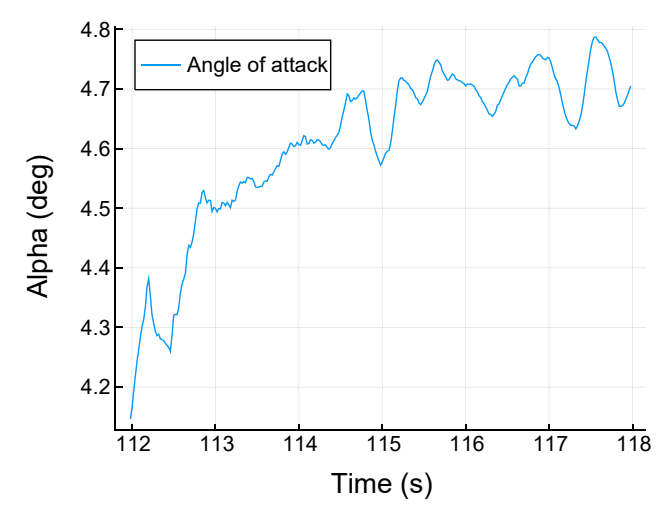

(a) $\alpha$ of flight data

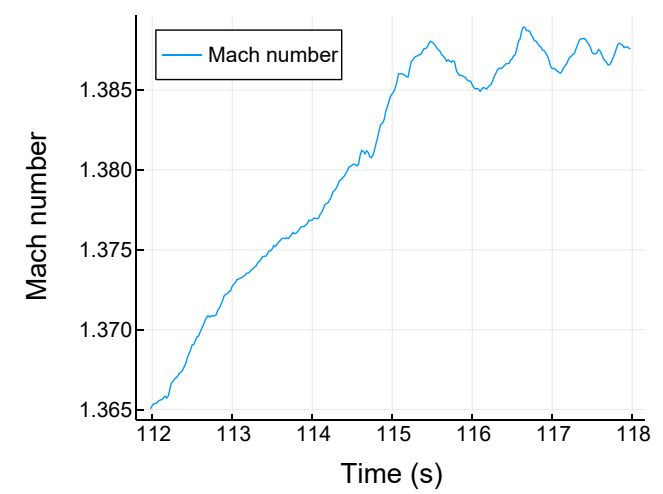

(b) $M$ of flight data

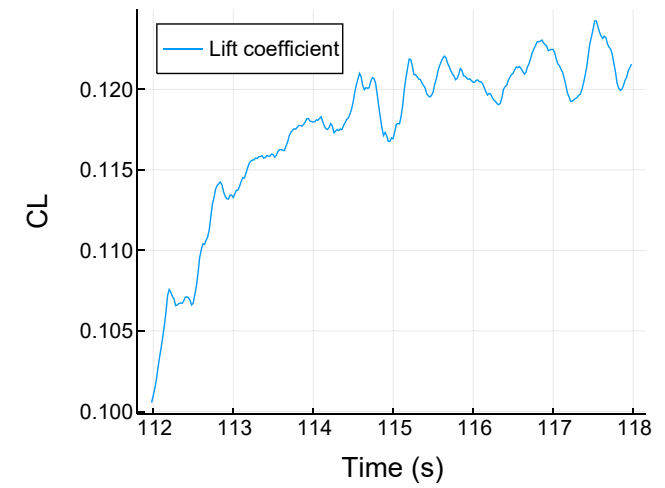

(c) $C_{L}$ of flight data

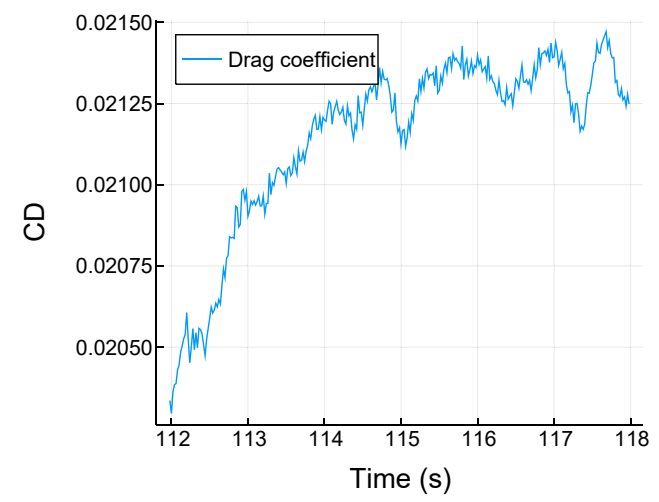

(d) $C_{D}$ of flight data

Fig. 6. Flight data. 
Trans. Japan Soc. Aero. Space Sci., Vol. 63, No. 6, 2020

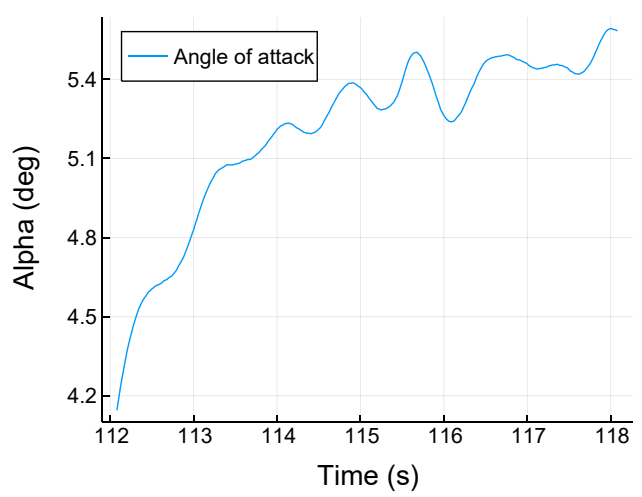

(a) $\alpha$ of simulation data

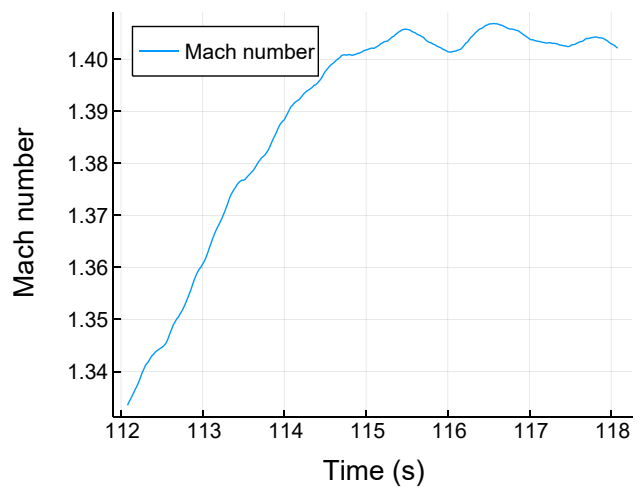

(b) $M$ of fsimulation data

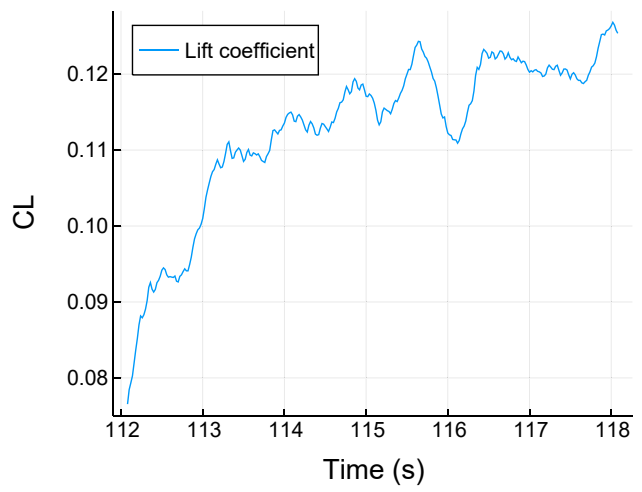

(c) $C_{L}$ of simulation data (noisy)

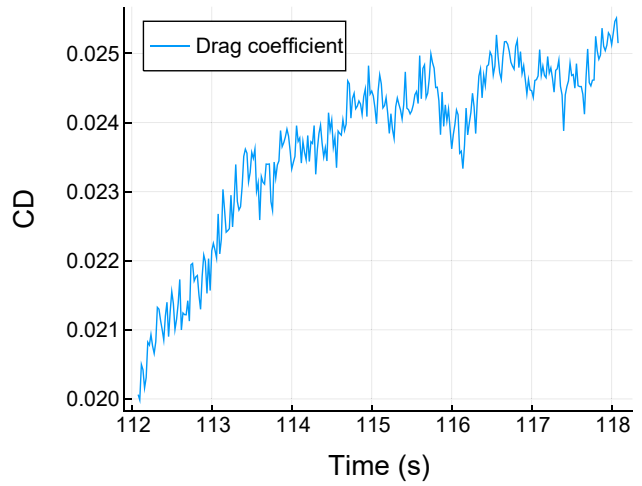

(d) $C_{D}$ of simulation data (noisy)

Fig. 7. Simulation data (noisy).

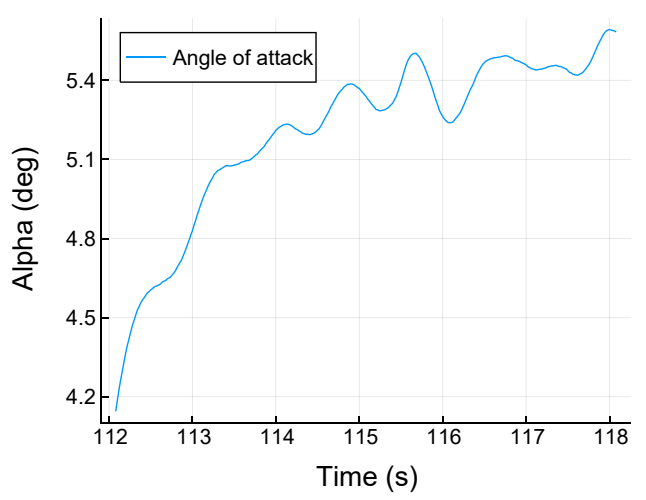

(a) $\alpha$ of simulation data

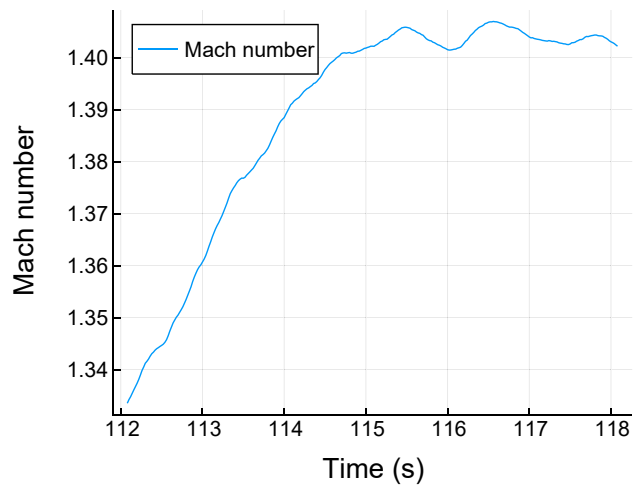

(b) $M$ of simulation data

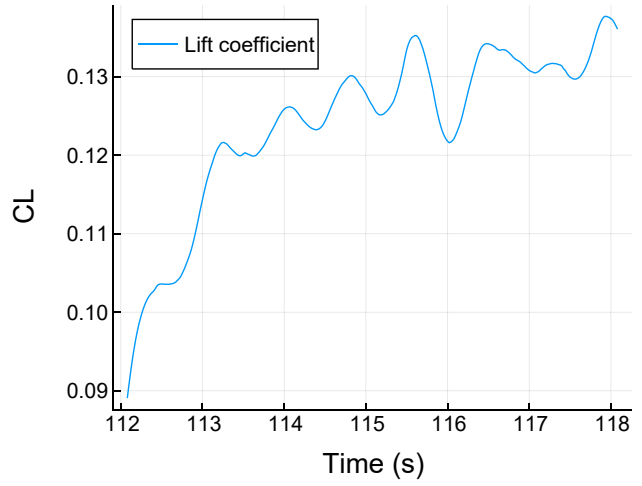

(c) $C_{L}$ of simulation data (noise-free)

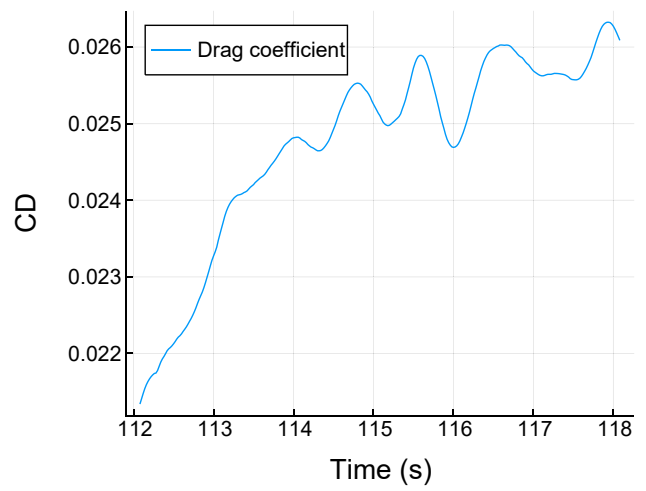

(d) $C_{D}$ of simulation data (noise-free)

Fig. 8. Simulation data (noise-free). 